\title{
THE RELATIVISTIC INCOMPRESSIBLE SPHERE
}

\author{
H. A. BUCHDAHL and W. J. LAND *
}

(Received 26 August 1966)

\begin{abstract}
Summary. The Schwarzschild Interior Solution represents a static sphere the proper density of which has the same value throughout. Though it is sometimes referred to as an "incompressible" sphere it is physically unacceptable since (formally) the speed of sound within it is infinite. Perhaps the most natural analogue of the classical incompressible sphere is therefore a sphere such that the speed of sound is everywhere just equal to the speed of light. This paper investigates spheres of this kind in some detail.
\end{abstract}

\section{Introduction}

The static spherically symmetric solution of the gravitational field equations generally known as the Schwarzschild Interior Solution is frequently said to represent an incompressible fluid sphere ${ }^{1}$, on the grounds that the total density $\rho\left(\equiv T_{4}^{4}\right)$ has a fixed value throughout the sphere. Unfortunately, $\rho$ being thus independent of the hydrostatic pressure $p$, the velocity of sound is everywhere infinite, so that this solution lacks physical meaning in the sense that it is certainly physically unrealizable. For this reason it is desirable to seek some alternative definition of an "incompressible fluid", but any such definition will have some degree of arbitrariness ${ }^{2}$. Now, in a Newtonian situation, incompressibility of any medium entails the possibility of transmitting a mechanical disturbance with infinite speed. It suggests itself therefore, to adopt the view that in a relativistic situation the speed of propagation of a sound wave (i.e., mechanical shock wave of sufficiently small amplitude) should be the largest possible permitted by the internal consistency of the theory, that is to say, the speed of light. Accordingly we here lay down that the equation of state of the fluid constituting the sphere shall be ${ }^{3}$

$$
p=\rho-\rho_{b},
$$

* Present Address: Physics Department, Harvard University, Cambridge, Mass.

1 E.g., R. C. Tolman, Relativity, Thermodynamics and Cosmology (Clarendon Press, Oxford, 1934), Chap. 7, p. 245.

2 See the discussion of Synge: J. L. Synge, Relativity: The Special Theory (North-Holland Publishing Company, Amsterdam, 1958), Chap. 8, $\$ 15$, p. 306.

3 Units are such that the speed of light $c$ and Newton's constant $k$ both take the numerical value unity. The subscripts $b$ and $c$ refer to the boundary and centre of the sphere respectively. 
since this relation entails that the velocity of sound is everywhere unity. The object of the present work is to investigate in some detail the consequences of adopting (1.1).

A second order differential equation which is central to the whole problem is derived in Section 2. It is solved in a series of ascending powers of the radial variable for sufficiently small values of the central density $\rho_{c}\left(\rho_{b} \neq 0\right)$, and under these conditions expressions are obtained for various quantities associated with the sphere, such as its field producing mass, its coordinate radius, and so on (Section 3 ). In Section 4 an exact but irregular solution of the central equation is presented, and in Section 5 this is made the basis for a heuristic investigation concerning the asymptotic form of the solution, valid for sufficiently large $\rho_{c}$. After some incidental remarks concerning certain known general inequalities relating to regular static spheres (Section 6), Sections 7 and 8 finally deal with the results obtained by numerical integration of the equations of the sphere.

\section{Basic equations}

(a) The field being static, static and spherically symmetric canonical coordinates may be chosen so that the metric takes the form

$$
d s^{2}=-e^{-\lambda(r)} d r^{2}-r^{2}\left(d \theta^{2}+\sin ^{2} \theta d \phi^{2}\right)+e^{\nu(r)} d t^{2} .
$$

The field equations are then 4

$$
\begin{aligned}
8 \pi r^{2} p & =e^{-\lambda}\left(r v^{\prime}+1\right)-1, \\
8 \pi r^{2} \rho & =e^{-\lambda}\left(r \lambda^{\prime}-1\right)+1, \\
p^{\prime} & =-\frac{1}{2}(\rho+p) \nu^{\prime},
\end{aligned}
$$

primes denoting derivatives with respect to $r$. It is convenient to use the notation introduced elsewhere, ${ }^{5}$ i.e., write

$$
P=4 \pi p, \quad x=r^{2}, \quad w=r^{-3} \int_{0}^{r} r^{2} v d r
$$

where

$$
v=4 \pi \rho \text {. }
$$

Then from (1.1) and I (2.11) it follows that

$$
2(1-2 x w)\left(2 x w{ }_{, x x}+5 w_{, x}\right)+\left(2 x w{ }_{, x}+4 w-v_{b}\right)\left(4 x w_{, x}+6 w-v_{b}\right)=0 .
$$

Taking $v_{b}$ not to be zero, write

$$
u=3 v_{b}^{-1} w, \quad z=\frac{1}{3} v_{b} x .
$$

- Reference 2, p. 245. The cosmical constant is taken to be zero.

b H. A. Buchdahl, Phys. Rev. 116 (1959), 1027. This paper will be referred to as I. 
Then, if dots denote derivatives with respect to $z,(2.9)$ gives rise to the central equation

(2.11) $\quad 4 z(1-2 z u) \ddot{u}+8 z^{2} \dot{u}^{2}+8 z u \dot{u}+2(5-9 z) \dot{u}+3(4 u-3)(2 u-1)=0$.

It may be noted that

$$
P=\frac{1}{3} v_{b}(2 z \dot{u}+3 u-3) .
$$

(b) The central value of $u$ is

$$
u_{\mathrm{c}}=1+\eta
$$

say, so that

$$
\eta=\rho_{c} / \rho_{b}-1>0,
$$

granted that the sphere is regular at the centre.

$$
\text { If } 3 p_{c} / \rho_{c}=\beta(<3) \text { (cf. I, Section 2) }
$$

$$
\eta=\beta /(3-\beta) \text {. }
$$

Consequently, if it be assumed, as is frequently done, that the trace of the energy-momentum tensor cannot be negative, one has $\beta \leqq 1$, i.e.,

$$
\eta \leqq \frac{1}{2}
$$

The physically interesting values of $\eta$ therefore appear to lie in the narrow range $0<\eta \leqq \frac{1}{2}$.

Let $M$ be the field producing mass of the sphere and $R$ its coordinate radius; and write

$$
\mu=M / R \text {. }
$$

Then from (2.10) and $I(2.17)$

$$
z_{\mathrm{b}} u_{\mathrm{b}}=\mu .
$$

The use of boundary conditions is already implicit in $I(2.17)$. They are also required in fixing the relation between $v$ and $v$ which follows from (1.1) and (2.4):

$$
e^{\nu}=(1-2 \mu)\left(2 v / v_{b}-1\right)^{-1}
$$

In particular

$$
\left(e^{\nu}\right)_{c}=(1-2 \mu) /(1+2 \eta)
$$

\section{Solution in series ( $\eta$ small)}

For sufficiently small values of $\eta$ the central equation (2.11) may be solved as a series in ascending powers of $\eta$. (In view of the complexity 
of the equation it will simply be assumed that for a certain range of values of $\eta$ the various series considered below do indeed converge: rapidly so if successive early terms of the series rapidly decrease in magnitude.) Some tedious work leads to the result

$$
\begin{aligned}
u= & (1+\eta)-\frac{3}{10}(1+2 \eta)(1+4 \eta) z+\frac{3}{35}(1+2 \eta)(1+4 \eta)(1+7 \eta) z^{2} \\
& -\frac{1}{105}(1+2 \eta)(1+4 \eta)\left(4+29 \eta+112 \eta^{2}\right) z^{3} \\
& +\frac{1}{3850}(1+2 \eta)(1+4 \eta)\left(-1+602 \eta+2440 \eta^{2}+6664 \eta^{3}\right) z^{4}+O\left(z^{5}\right) .
\end{aligned}
$$

The pressure vanishes at the boundary. Using (3.1) in (2.12) one therefore gets an algebraic equation which determines $z_{b}$ as a function of $\eta$. It turns out that

$$
z_{b}=2 \eta\left(1-\frac{26}{5} \eta+\frac{4264}{175} \eta^{2}-\frac{57752}{525} \eta^{3}\right)+O\left(\eta^{5}\right) .
$$

It will be observed that the ratio of successive terms of this series is of the order of 5. One therefore cannot expect the first few terms of the series for $z_{b}$ to be a useful approximation unless $z_{b}$ is less than of the order of $\frac{1}{10}$, say. Using (3.2) in (3.1) one can now go on to express $u_{b}$ as a function of $\eta$. With (2.17) one thus gets

$$
\mu=2 \eta\left(1-\frac{24}{5} \eta+\frac{3876}{175} \eta^{2}-\frac{3488}{35} \eta^{3}\right)+O\left(\eta^{5}\right) .
$$

From these results one deduces that

$$
M=\frac{4}{3} \pi \rho_{b} R^{3}\left(1+\frac{1}{5} \mu+O\left(\mu^{2}\right)\right),
$$

whereas for the Schwarzschild sphere the factor in brackets is of course absent. Inverting (3.3),

$$
\eta=\frac{1}{2} \mu\left(1+\frac{12}{5} \mu+\frac{1047}{175} \mu^{2}+\frac{2684}{175} \mu^{3}\right)+O\left(\mu^{5}\right),
$$

one has a relation which allows one to replace $\eta$ everywhere by the directly observable quantity $\mu$. (Recall that the gravitational redshift is given immediately by the value of the quantity

$$
\Delta \equiv\left(g_{44}\right)_{b}=1-2 \mu \text {, }
$$

(cf. I Section 1).)

The (negative) gravitational potential energy of the sphere is (cf. I Section 6)

$$
\Omega=M_{0}-M=\int_{0}^{R}\left(e^{\frac{1}{2} \lambda}-1\right) v r^{2} d r
$$

One thus has to evaluate the integral

$$
\frac{1}{2}\left(3 / v_{b}\right)^{\frac{1}{2}} \int_{0}^{x_{b}}\left[(1-2 z u)^{-\frac{1}{2}}-1\right](2 z \dot{u}+3 u) z^{\frac{1}{2}} d z .
$$


It turns out that

$$
\Omega=\left(3 M^{2} / 5 R\right)\left(1+\frac{11}{10} \mu+\frac{3}{2} \mu^{2}+O\left(\mu^{3}\right)\right) .
$$

Another quantity of interest is the physical radius

$$
R^{*}=\int_{0}^{R} e^{\frac{1}{2} \lambda} d r
$$

One finds

$$
R^{*}=R\left(1+\frac{1}{3} \mu+\frac{17}{50} \mu^{2}+O\left(\mu^{3}\right)\right)
$$

\section{An exact irregular solution}

It may be confirmed by direct substitution that $(2.11)$ has the following exact solution:

$$
u=\frac{1}{4} z^{-1}+\frac{1}{2}
$$

(4.1) violates the condition of regularity at the centre in as far as the pressure and density become infinite at the centre whilst $g_{44}$ vanishes there. On the other hand $e^{\lambda}$ remains finite there, whilst $M, R, \cdots$, etc., are also finite. Still taking $v_{b} \neq 0$ (since otherwise $R$ and $M$ will be infinite), the units used here may be further restricted by arranging $v_{b}$ also to take the numerical value unity. Then one has in the first place

(4.2-5) $\quad P=\frac{1}{12} z^{-1}-\frac{1}{2}, \quad v=\frac{1}{12} z^{-1}+\frac{1}{2}, \quad e^{-\lambda}=\frac{1}{2}-z, \quad e^{\nu}=2 z$.

(4.2) and (4.4) show that

$$
z_{b}=\frac{1}{6}, \quad \mu=\frac{1}{3}
$$

Then, in view of $(2.10)$,

$$
M=\frac{1}{3} \sqrt{ } 2, \quad R=1 / \sqrt{ } 2 .
$$

The evaluation of $R^{*}$ and $\Omega$ leads to elementary integrals with the results

$$
\begin{aligned}
R^{*} & =\sqrt{ } 3 \arcsin (1 / \sqrt{ } 3) \\
\Omega & =\frac{1}{24}[15 \sqrt{ } 3 \arcsin (1 / \sqrt{ } 3)-3 \sqrt{ } 6-4 \sqrt{ } 2] .
\end{aligned}
$$

\section{Heuristic considerations concerning the limit $\eta \rightarrow \infty$}

The exact solution presented in the preceding section gives an infinite central value of $u$, whatever the value of $v_{b}$ may be. Presumably the solution of (2.11) corresponding to a finite, sufficiently large, value of $u_{c}$ will be closely approximated by (4.1) except in a certain neighbourhood of the origin. To investigate this situation write 


$$
\varepsilon=1 / u_{c} \quad\left(=v_{b} / v_{c}\right),
$$

and set

$$
u=u_{c} U, \quad z=s / u_{c} .
$$

In terms of the original variables therefore

$$
U=w / w_{c}, \quad s=w_{c} x .
$$

Then (2.11) becomes

$$
\begin{gathered}
4 s(1-2 s U) U^{\prime \prime}+8 s^{2} U^{\prime 2}+8 s U U^{\prime}+10 U^{\prime}+24 U^{2} \\
-\varepsilon\left(18 s U^{\prime}+30 U\right)+9 \varepsilon^{2}=0,
\end{gathered}
$$

where primes now denote derivatives with respect to $s$. Note that

$$
P=w_{c}\left(2 s U^{\prime}+3 U-3 \varepsilon\right) .
$$

The exact solution (4.1) becomes

$$
U=\frac{1}{4} s^{-1}+\frac{1}{2} \varepsilon
$$

in the present variables.

We now make the heuristic assumption that for sufficiently large $s$ the required solution of (5.4) has the form

$$
U \sim \frac{1}{4} s^{-1}+\frac{1}{2} \varepsilon+\Gamma,
$$

where the function $\Gamma$ satisfies the equation which arises from the substitution of (5.7) into (5.4) when only terms linear in $\Gamma$ and its derivatives are retained. This equation turns out to be

$$
(1-2 \varepsilon s) s^{2} \Gamma^{\prime \prime}+(4-7 \varepsilon s) \Gamma^{\prime}+3(1-\varepsilon s) \Gamma=0 .
$$

It has the solution

$$
\begin{gathered}
\Gamma=A^{\dagger} \operatorname{Re} G+B^{\dagger} \operatorname{Im} G, \\
G=s^{n} F\left(n+1, n+\frac{3}{2} ; 2 n+4 ; 2 \varepsilon s\right), \quad\left(n=-\frac{3}{2}+\frac{1}{2} i \sqrt{ } 3\right),
\end{gathered}
$$

where $A^{\dagger}$ and $B^{\dagger}$ are constants. (The hypergeometric function which occurs here is in fact an elementary algebraic function of $s$.)

The case $\varepsilon=0$ corresponds to finite central density but zero boundary density. (The "boundary" occurs at infinity.) For all finite $s$ one then has

$$
\Gamma=A_{0} \operatorname{Re} s^{n}+B_{0} \operatorname{Im} s^{n},
$$

where $A_{0}=A_{\varepsilon=0}^{\dagger}$, etc.

In view of (5.5)

$$
P=w_{c}\left(\frac{1}{4} s^{-1}-\frac{3}{2} \varepsilon+\Gamma^{*}\right),
$$

with

$$
\Gamma^{*}=2 s \Gamma^{\prime}+3 \Gamma
$$


so that the boundary value $s_{b}$ of $s$ is the first positive zero of the right hand member of (5.11). To progress beyond this point we now restrict $\varepsilon$ to be small. When $\varepsilon$ is sufficiently small one deduces from (5.9) that

$$
\Gamma^{*}(s=1 / 6 \varepsilon)=O\left(\varepsilon^{\frac{3}{2}}\right\rangle,
$$

granted that $A$ and $B$ are continuous functions of $\varepsilon$ in a neighbourhood of the point $\varepsilon=0$, at which their values are $A_{0}, B_{0}$ (cf. (5.10)). Then from (5.11)

$$
s_{b}=1 / 6 \varepsilon+O\left(\varepsilon^{\frac{1}{2}}\right) .
$$

Inserting (5.13) in (5.9) one gets

$$
G_{b}=(6 \varepsilon)^{-n}\left[F\left(n+1, n+\frac{3}{2} ; 2 n+4 ; \frac{1}{3}\right)+O\left(\varepsilon^{\frac{3}{2}}\right)\right] .
$$

When $\varepsilon$ is sufficiently small the term $O\left(\varepsilon^{\frac{3}{2}}\right)$ may be discarded and $A^{\dagger}$, $B^{\dagger}$ replaced by $A_{0}, B_{0}$ respectively. In this way one arrives at a result of the general form

$$
\Gamma_{b} \sim A \varepsilon^{\frac{3}{2}} \sin (\tau+\alpha),
$$

where $A$ and $\alpha$ are certain constants and

$$
\tau=\frac{1}{2} \sqrt{ } 3 \ln (1 / \varepsilon) .
$$

$\Gamma_{b} *$ may be evaluated after the same fashion. Thus generically

$$
\Gamma_{b}^{*} \sim 3 \sqrt{ } 2 B \varepsilon^{\frac{3}{2}} \sin (\tau+\beta),
$$

where $B$ and $\beta$ are certain constants related to $A$ and $\alpha$. However, the relationships in question are so complicated that we do not consider them explicitly here.

(5.11) and (5.16) now yield

whence

$$
z_{b} \sim \frac{1}{6}(1+2 \sqrt{ } 2 B \sqrt{ } \varepsilon \sin (\tau+\beta))
$$

$$
R \sim \frac{1}{\sqrt{ } \mathbf{2}}+B \sqrt{ } \varepsilon \sin (\tau+\beta) .
$$

Again, since $M=s_{b} U_{b} R$ it follows from (5.17) and (5.14) that

$$
M \sim \frac{1}{3 \sqrt{ } \mathbf{2}}+C \sqrt{ } \varepsilon \sin (\tau+\gamma),
$$

where $c$ and $\gamma$ are related to the constants introduced earlier. (If $R$ and $M$ are required in $c g s$ units factors $\left(c^{2} / 4 \pi k \rho_{b}\right)^{\frac{1}{2}}$ and $\left(c^{6} / 4 \pi k^{3} \rho_{b}\right)^{\frac{1}{2}}$ have to be supplied on the right of (5.17) and (5.18) respectively.)

According to $(5.17,18)$ one has for sufficiently small values of $\varepsilon$ a curve in the $R-M$ plane which spirals around the limiting point $\frac{1}{3}$. If 
the line joining this point to points in the spiral has a certain direction when $\varepsilon=\varepsilon^{\prime}$, and, as $\varepsilon$ increases, it first resumes this direction when $\varepsilon=\varepsilon^{\prime \prime}$, then

$$
\varepsilon^{\prime \prime} / \varepsilon^{\prime}=e^{4 \pi / \sqrt{ } 3} \approx 1415.4
$$

\section{Concerning certain inequalities}

Some general inequalities which appear in I were subsequently strengthened in a paper ${ }^{6}$ hereafter referred to as II. These gave some information about the spheres under review without limitation on the values of $\eta$. However, for general values of this parameter the inequalities in question are algebraically somewhat involved. Accordingly we restrict ourselves to the case $\eta=\frac{1}{2}$ (i.e., $\beta=1$ ) this device being motivated partly by (2.15) and partly by the fact that when $\eta=\frac{1}{2}$ neither the series expansions of Section 3 nor the asymptotic results of Section 5 are of any use. The inequalities to be considered are II(4.10) and II(5.9) which read

$$
\Delta>\frac{2}{9}(1+\delta)
$$

and

$$
\Delta<1-\frac{5}{9} \delta^{\frac{1}{3}}
$$

in the present context. Here $\delta=2 M / R^{3}$, so that (6.2) gives

$$
M>5 \sqrt{ } 5 / 54 \approx 0.2070,
$$

whilst (6.1) becomes

$$
M<7 R^{3} /\left(18 R^{2}+4\right) .
$$

(6.3) and (6.4) together give rise to the further inequality

i.e., approximately,

$$
189 R^{3}-45 \sqrt{ } 5 R-10 \sqrt{ } 5>0,
$$

$$
R>0.82236 .
$$

It is worthy of note that the number on the right is greater than $R_{\infty}=1 / \sqrt{ } 2$.

\section{Numerical results}

Equation (2.11) was integrated by numerical methods on an IBM 1620 computer. The work was carried out for a reasonably large selection of values of $\eta$. It will suffice here to present an extract of the results obtained, in the form of Table I which gives $P$ as a function of $r$ for a few

- H. A. Buchdahl, Astrophysical Journal, 146 (1966), 275. 
TABLE I

$P$ as function of $r$

\begin{tabular}{|c|c|c|c|c|c|c|c|c|c|c|}
\hline$r$ & $\eta \rightarrow 0.01$ & 0.04 & 0.1 & 0.2 & 0.5 & 1 & 4 & 9 & 19 & 99 \\
\hline 0 & .0100000 & .040000 & .100000 & .20000 & .50000 & 1.00000 & 4.00000 & 9.00000 & 19.0000 & 99.0000 \\
\hline .05 & .0095582 & .039478 & .099300 & .19895 & .49750 & .99377 & 3.93686 & 8.17323 & 17.8027 & 72.4646 \\
\hline .10 & .0082345 & .037916 & .097206 & .19581 & .49006 & .97526 & 3.75456 & 7.92177 & 14.7737 & 34.6386 \\
\hline .15 & .0060347 & .035320 & .093732 & .19062 & .47780 & .94507 & 3.47285 & 6.80034 & 11.1170 & 15.2861 \\
\hline .20 & .0029680 & .031705 & .088901 & .18341 & .46094 & .90412 & 3.11991 & 5.55634 & 7.82439 & $\mathbf{7 . 4 4 6 0 3}$ \\
\hline .25 & & .027087 & .082744 & .17427 & .43977 & .85363 & 2.72696 & 4.36136 & 5.30740 & 4.08621 \\
\hline .30 & & .021492 & .075304 & .16326 & .41465 & .79503 & 2.32316 & 3.31858 & 3.54850 & 2.45369 \\
\hline .35 & & .014944 & .066627 & .15050 & .38599 & .72989 & 1.93210 & 2.46603 & 2.36819 & 1.56164 \\
\hline .40 & & .007476 & .056767 & .13609 & .35423 & .65985 & 1.57023 & 1.79804 & 1.58294 & 1.02598 \\
\hline .45 & & & .045787 & .12016 & .31984 & .58652 & 1.24698 & 1.28778 & 1.05514 & .67988 \\
\hline .50 & & & .033750 & .10283 & .28333 & .51142 & .96594 & .90283 & .69317 & .44311 \\
\hline .55 & & & .020726 & .08426 & .24515 & .43595 & .72652 & .61334 & .43884 & .27368 \\
\hline .60 & & & .006787 & .06456 & .20580 & .36132 & .52555 & .39493 & .25561 & .14797 \\
\hline .65 & & & & .04390 & .16571 & .28855 & .35855 & .22891 & .12038 & .05193 \\
\hline .70 & & & & .02240 & .12528 & .21844 & .22061 & .10144 & .01832 & \\
\hline .75 & & & & .01939 & .08490 & .15161 & .10705 & .00247 & & \\
\hline .80 & & & & & .04488 & .08847 & .01360 & & & \\
\hline .85 & & & & & .00548 & .02928 & & & & \\
\hline
\end{tabular}

values of $\eta$. (The step length of 0.05 does not correspond to that used in the course of computation when it was 0.01 in some cases and 0.005 in others. The number of significant figures has also been reduced.) Other functions of interest are easily obtained from Table I, if necessary by an elementary integration. For example,

$$
e^{\nu}=\Delta /(2 P+1), \quad w=r^{-3} \int_{0}^{r} r^{2} P d r+\frac{1}{3},
$$

and so on. (As regards $\Delta$, see Table II.)

The coordinate radius $R$ (and, consequently, quantities like $M, R^{*}$, etc.) cannot be read off from Table I. For each value of $\eta$ the step length was, in the course of computation, reduced further and further as the boundary was approached until with a step length $<10^{-7}$ a value of $r$ was arrived at for which $P<10^{-9}$. This value of $r$ was taken to be $R$. Table II gives the values of certain parameters associated with the spheres for various values of $\eta$.

It will be noted that $\delta \approx 0.78$ when $\eta$ has the largest value allowed by (2.15). In this case the solution evidently differs already fairly widely from the Schwarzschild Interior Solution.

To compare the results of Section 5 with those obtained by purely 
TABLE II

$R, R^{*}, M, \Omega, \delta, \Delta$ as functions of $\eta$

\begin{tabular}{rrrrrrr}
\hline \multicolumn{1}{r}{$\eta$} & $R$ & $R^{*}$ & \multicolumn{1}{c}{$M$} & $\Omega$ & $\delta$ & $\Delta$ \\
\hline 0.01 & .238788 & .24034 & .0045568 & .04534 & .9940 & .96184 \\
0.02 & .329534 & .33370 & .0120231 & $.00^{2} 2743$ & .9882 & .92703 \\
0.04 & .444973 & .45567 & .0298317 & .001297 & .9767 & .86592 \\
0.06 & .522017 & .53998 & .0483071 & .003025 & .9656 & .81406 \\
0.08 & .578984 & .60439 & .0667074 & .005308 & .9547 & .76957 \\
0.1 & .623294 & .65607 & .0838287 & .007999 & .9442 & .73101 \\
0.2 & .750431 & .81616 & .151331 & .024029 & .8952 & .59668 \\
0.3 & .808671 & .90024 & .195204 & .039870 & .8518 & .51722 \\
0.4 & .839581 & .95145 & .224525 & .053581 & .8130 & .46515 \\
0.5 & .857036 & .98518 & .244853 & .065053 & .7779 & .42860 \\
1 & .876076 & 1.05362 & .288551 & .099682 & .6437 & .34126 \\
4 & .808156 & 1.06059 & .293441 & .13414 & .3336 & .27380 \\
9 & .751419 & 1.03212 & .271559 & .13370 & .1920 & .27721 \\
19 & .710576 & 1.01039 & .250662 & .12808 & .1048 & .29448 \\
99 & .683262 & 1.01208 & .226652 & .11820 & .0213 & .33656 \\
500 & .702602 & 1.04899 & .231054 & .11988 & .0040 & .34229 \\
3000 & .711184 & 1.06590 & .237084 & .12404 & .0007 & .33327 \\
$\infty$ & .707107 & 1.06604 & .235702 & .12439 & 0 & .33333 \\
\hline
\end{tabular}

numerical work the case $\varepsilon=0$ may be considered first. According to (5.10) $\Gamma$ has then the form

$$
\Gamma=A s^{-\frac{3}{2}} \sin \left(\frac{1}{2} \sqrt{ } 3 \ln s+\alpha\right),
$$

where $A$ and $\alpha$ are constants of integration. Eq. (5.4) was integrated numerically for values of $s$ up to 1405. By inspection of the results obtained one finds that the largest zero less than 1405 of

$$
D=s^{\frac{3}{2}}(U-1 / 4 s)
$$

occurs at $s_{1} \approx 1103.3$, and here $D$ is increasing. As $s$ decreases from $s_{1}$ a minimum of $D$ first occurs at $s_{2} \approx 179.47$. Thus $s_{1} / s_{2} \approx 6.1475$, whereas (7.2) implies the value $\exp (\pi / \sqrt{ } 3) \approx 6.1337$ for this ratio, and this result is very satisfactory. The next zero of $D$ occurs at $s_{3} \approx 28.121$, whereas $s_{1} \exp (-2 \pi / \sqrt{ } 3) \approx 29.326$, again a satisfactory result. More generally, from the calculated values $s_{1}=1103.3$ and $D\left(s_{2}\right)=-0.04299$ one can obtain approximate values of $A$ and $\alpha$, viz.

$$
A=0.0430, \quad \alpha=-1.336 .
$$

One may also fit the results contained in Table II to eqs. (5.17). It turns out that with the values 
(7.5) $\quad B=0.245, \quad \beta=0.495 ; \quad c=0.128, \quad \gamma=-0.0185$

good agreement may be obtained. In fact the range of values of $\eta$ over which an adequate fit may be achieved is surprisingly large when one considers that $s$ has values less than of the order of $1 / 6 \varepsilon$ in the region in which $P \nless 0$.

As regards the inequalities of Section $6,(6.3,4)$ now yield $0.2070<M<0.2559$, where the computed value of $R$ has been used to calculate the upper bound. The actual value of $M$ lies quite close to the latter, as might have been expected, since when $\delta$ is not too small (6.1) is generally a strong inequality. Finally, the actual value 0.8570 of $R$ also lies quite close to the lower bound which appears in (6.5).

Australian National University Canberra 